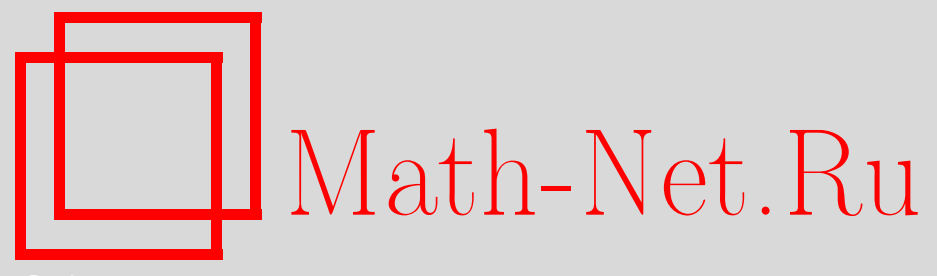

Е. В. Черепанова, Скорость приближения распределения числа циклов заданной длины случайной подстановки с известным числом циклов к предельным законам, Дискрет. матем., 2006, том 18, выпуск 3, 61-76

DOI: https://doi.org/10.4213/dm59

Использование Общероссийского математического портала Math-Net.Ru подразумевает, что вы прочитали и согласны с пользовательским соглашением http://www . mathnet.ru/rus/agreement

Параметры загрузки:

IP : 44.207 .124 .84

26 апреля 2023 г., $17: 14: 35$ 


\title{
Скорость приближения распределения числа циклов заданной длины случайной подстановки с известным числом циклов к предельным
} законам

\author{
() 2006 г. Е. В. Черепанова
}

\begin{abstract}
Рассматривается множество $S_{n, N}$ всех различных подстановок степени $n$, имеющих $N$ циклов, на котором задано равномерное распределение вероятностей. Для распределения числа циклов заданной длины случайной подстановки из $S_{n, N}$ получены оценки скорости сходимости к предельным распределениям, когда $n, N \rightarrow \infty$ так, что $n / N \rightarrow 1$ или $n / N \rightarrow \infty$.

Работа выполнена при поддержке Российского фонда фундаментальных исследований, проект 05-01-00007-а, и программой Президента Российской Федерации поддержки ведущих научных школ, грант НШ 1758.2003.1.
\end{abstract}

В [1] рассматривалась классическая схема размещения $n$ частиц по $N$ ячейкам и были получены предельные распределения случайной величины $\mu_{r}(n, N)$, равной числу ячеек, содержащих ровно по $r$ частищ, при различном характере стремления $n, N$ к бесконечности. В частности, доказано, что если $r \geqslant 2$ фиксировано и выполнено одно из условий $n / N \rightarrow 0, n^{r} / r ! N^{r-1} \rightarrow \infty$ или $n / N \rightarrow \infty, \mathrm{E} \mu_{r}(n, N) \rightarrow \infty$, то распределения $\mu_{r}(n, N)$ сближаются одновременно с нормальным законом и распределением Пуассона. В указанных областях изменения параметров $n, N$ получены оценки близости распределения $\mu_{r}(n, N)$ к этим законам, и указано, какое приближение является более точным в зависимости от значения параметра $n / N$. Аналогичная задача оценки скорости сходимости к предельным распределениям возникает для случайных подстановок с известным числом циклов.

Пусть на множестве $S_{n, N}$ всех различных подстановок степени $n$, имеющих $N$ циклов, задано равномерное распределение вероятностей. Обозначим через $\mu_{r}$ случайную величину, равную числу циклов длины $r$ в случайно выбранной подстановке из $S_{n, N}$. В $[2,3]$ изучалось предельное поведение $\mu_{r}$ при $n, N \rightarrow \infty$. В [3] доказано, что в некоторых зонах изменения параметров $n, N, r$ имеет место сходимость распределения $\mu_{r}$ одновременно к нормальному закону и к распределению Пуассона. В данной статье в теоремах 1, 2 получены оценки скорости сходимости распределения $\mu_{r}$ к предельным законам при $n / N \rightarrow 1$ и $n / N \rightarrow \infty$, в теоремах 3,4 найдены расстояния по вариации между распределением $\mu_{r}$ и предельными законами, а в следствиях 1-4 указано, какое из двух приближений лучше. 
Введем независимые одинаково распределенные случайные величины $\xi_{1}, \ldots, \xi_{N}$ и $\xi_{1}^{(r)}, \ldots, \xi_{N}^{(r)}$ такие, что

$$
\begin{aligned}
p_{k}=\mathbf{P}\left\{\xi_{1}=k\right\} & =\frac{\lambda^{k}}{-k \ln (1-\lambda)}, & 0<\lambda<1, & k=1,2, \ldots, \\
\mathbf{P}\left\{\xi_{1}^{(r)}=k\right\} & =\mathbf{P}\left\{\xi_{1}=k \mid \xi_{1} \neq r\right\}, & & k=1,2, \ldots
\end{aligned}
$$

В [4] показано, что для $k=0,1, \ldots, N$ при любом значении параметра $\lambda$ распределения (1) справедливо равенство

$$
\mathbf{P}\left\{\mu_{r}=k\right\}=\left(\begin{array}{c}
N \\
k
\end{array}\right) p_{r}^{k}\left(1-p_{r}\right)^{N-k} \frac{\mathbf{P}\left\{\zeta_{N-k}^{(r)}=n-k r\right\}}{\mathbf{P}\left\{\zeta_{N}=n\right\}},
$$

где

$$
\begin{aligned}
\zeta_{N} & =\xi_{1}+\ldots+\xi_{N}, \\
\zeta_{N-k}^{(r)} & =\xi_{1}^{(r)}+\ldots+\xi_{N-k}^{(r)} .
\end{aligned}
$$

Следуя [1], введем обозначения

$$
\begin{aligned}
\Pi(k, \alpha) & =\frac{\alpha^{k}}{k !} e^{-\alpha}, \\
N\left(k, a, b^{2}\right) & =\frac{1}{b \sqrt{2 \pi}} \exp \left\{-\frac{(k-a)^{2}}{2 b^{2}}\right\}, \\
\beta & =\frac{(m-r)^{2}}{\sigma^{2}} p_{r}, \\
\sigma_{r r}^{2} & =p_{r}\left(1-p_{r}-\beta\right),
\end{aligned}
$$

где

$$
\begin{aligned}
m & =\mathbf{E} \xi_{1}=-\frac{\lambda}{(1-\lambda) \ln (1-\lambda)} \\
\sigma^{2} & =\mathbf{D} \xi_{1}=\frac{m}{1-\lambda}-m^{2}
\end{aligned}
$$

Будем считать далее, что параметр $\lambda$ распределения (1) равен единственному решению уравнения

$$
-\frac{\lambda}{(1-\lambda) \ln (1-\lambda)}=\frac{n}{N}
$$

из интервала $(0,1)$. Через $C, C_{1}, C_{2}, \ldots$ будем обозначать положительные постоянные, не всегда различные. Справедливы следующие утверждения.

Теорема 1. Пусть $n, N \rightarrow \infty$ так, что $N p_{r} \rightarrow \infty$, и выполнено одно из следующих условий:

(a) $n / N \rightarrow 1, r \geqslant 3$;

(б) $n / N \rightarrow \infty$; 
u nycmb

$$
\left(\beta+p_{r}\right) v_{r}^{2} \rightarrow 0
$$

где

$$
v_{r}=\frac{k-N p_{r}}{\sqrt{N p_{r}}}
$$

Тогда

$$
\begin{aligned}
\mathbf{P}\left\{\mu_{r}=k\right\}=\Pi\left(k, N p_{r}\right)\left(1+\frac{\beta+p_{r}}{2}\left(1-v_{r}^{2}\right)\right) & \\
& \left.+O\left(\left(\beta^{2}+p_{r}^{2}\right)\left(1+v_{r}^{4}\right)+\beta p_{r} v_{r}^{2}\right)+R_{1}\right),
\end{aligned}
$$

где

$$
R_{1}= \begin{cases}o\left(\frac{1}{\sqrt{n-N}}\right), & \text { если } n / N \rightarrow 1 \\ o\left(\sqrt{\frac{\ln (n / N)}{N}}\right), & \text { если } n / N \rightarrow \infty\end{cases}
$$

Теорема 2. Пусть $n, N \rightarrow \infty$, выполнено одно из условий (а), (б) теоремы $1, u$

$$
\frac{1+u_{r}^{6}}{N p_{r}} \rightarrow 0, \quad\left(\beta+p_{r}\right) u_{r}^{2} \leqslant C<\infty,
$$

где

$$
u_{r}=\frac{k-N p_{r}}{\sigma_{r r} \sqrt{N}}
$$

Тогда

$$
\begin{aligned}
\mathbf{P}\left\{\mu_{r}=k\right\}=N(k, & \left.N p_{r}, N \sigma_{r r}^{2}\right) \\
& +\left(1+\frac{3 u_{r}-u_{r}^{3}}{6 \sqrt{N p_{r}}}+O\left(\frac{\left|u_{r}\right|+\left|u_{r}\right|^{3}}{\sqrt{N p_{r}}}\left(\beta+p_{r}\right)+\frac{1+u_{r}^{6}}{N p_{r}}\right)+R_{2}\right),
\end{aligned}
$$

гde

$$
R_{2}= \begin{cases}o\left(\frac{1}{\sqrt{n-N}}\right), & \text { если } n / N \rightarrow 1 \\ o\left(\left(1+\left|u_{r}\right|\right) \sqrt{\frac{\ln (n / N)}{N}}\right), & \text { если } n / N \rightarrow \infty\end{cases}
$$

Введем расстояния по вариации (см. [5]) между распределением $\mu_{r}$ и аппроксимирующими распределениями:

$$
\begin{aligned}
& \rho_{1 r}=\sum_{k=0}^{N}\left|\mathbf{P}\left\{\mu_{r}=k\right\}-\Pi\left(k, N p_{r}\right)\right|, \\
& \rho_{2 r}=\sum_{k=0}^{N}\left|\mathbf{P}\left\{\mu_{r}=k\right\}-N\left(k, N p_{r}, N \sigma_{r r}^{2}\right)\right| .
\end{aligned}
$$


Следуя [1], положим

$$
\begin{aligned}
& c_{1}=\frac{1}{2 \sqrt{2 \pi}} \int_{-\infty}^{\infty}\left|1-u^{2}\right| e^{-u^{2} / 2} d u=\sqrt{\frac{2}{\pi e}} \\
& c_{2}=\frac{1}{6 \sqrt{2 \pi}} \int_{-\infty}^{\infty}\left|u^{3}-3 u\right| e^{-u^{2} / 2} d u=\frac{1+4 e^{-3 / 2}}{3 \sqrt{2 \pi}}
\end{aligned}
$$

Теорема 3. Если $n, N \rightarrow \infty$ так, что $N p_{r} \rightarrow \infty$ и выполнено одно из условий (а), (б) теоремы 1, то

$$
\rho_{1 r}=c_{1}\left(\beta+p_{r}\right)+o\left(\beta+p_{r}\right)+R_{1},
$$

где $R_{1}$ определено в (8).

Теорема 4. Если $n, N \rightarrow \infty$ так, что $N p_{r} \rightarrow \infty$ и выполнено одно из условий (а), (б) теоремы 1, то

$$
\rho_{2 r}=\frac{c_{2}}{\sqrt{N p_{r}}}+o\left(\frac{1}{\sqrt{N p_{r}}}+\sqrt{\beta e^{-1 / \beta}}+\sqrt{p_{r} e^{-1 / p_{r}}}\right) .
$$

Замечание 1. Из доказательств теорем 1-4 (см. ниже) ясно, что утверждения этих теорем справедливы и в случае $r \rightarrow \infty$.

Следствие 1. Пусть $r \geqslant 3, n, N \rightarrow \infty$ так, что $n / N \rightarrow 1, N p_{r} \rightarrow \infty$. Если $\beta=O(1 / \sqrt{n-N})$, то лучшее приближение для распределения $\mu_{r}$ дает закон Пуассона, а если $1 / N p_{r}=o\left(\beta e^{-1 / \beta}\right)$ u $1 / \sqrt{n-N}=o(\beta)$, то лучшим приближением для распределения $\mu_{r}$ является нормальный закон.

Следствие 2. Если $r \geqslant 3$ фиксировано, $n, N \rightarrow \infty$ так, что $n / N \rightarrow 1, N p_{r} \rightarrow \infty$, $1 / \sqrt{n-N}=o(\beta)$ и $\beta e^{-1 / \beta}=O\left(1 /\left(N p_{r}\right)\right)$, то величины $\rho_{1 r}, \rho_{2 r}$ асимптотически равны при $\lambda=\lambda_{1 r}$, гдe

$$
\lambda_{1 r}=\left(\frac{c_{2}^{2} r^{3}}{4 c_{1}^{2}(1-r)^{4} N}\right)^{1 /(3 r-5)}(1+o(1)) .
$$

В этом случае лучшее приближение для распределения $\mu_{r}$ при $\lambda<\lambda_{1 r}$ дает распределение Пуассона, при $\lambda>\lambda_{1 r}-$ нормальный закон, а если $\lambda=\lambda_{1 r}$, то расстояния между распределением $\mu_{r}$ и приближаючими распределениями асимптотически равны

$$
\rho_{1}=\left(2 c_{1} c_{2}^{2}(1-r)^{2}\right)^{1 / 3}\left(\frac{4 c_{1}^{2}(1-r)^{4}}{c_{2}^{2} r^{3} N^{3 r-6}}\right)^{1 /(9 r-15)}(1+o(1)) .
$$

Следствие 3. Пусть $r$ фиксировано, $n, N \rightarrow \infty$ так, что $n / N \rightarrow \infty, N p_{r} \rightarrow \infty$. Если $p_{r}=o(\sqrt{\ln (n / N) / N})$, то лучшим приближением для распределения $\mu_{r}$ является закон Пуассона, а если $\sqrt{\ln (n / N) / N}=O\left(p_{r}\right), 1 / N p_{r}=o\left(p_{r} e^{-1 / p_{r}}\right)$, то более точное приближение для распределения $\mu_{r}$ дает нормальный закон.

Следствие 4. Пусть $r$ фиксировано, $n, N \rightarrow \infty$ так, что $n / N \rightarrow \infty, N p_{r} \rightarrow \infty$. При $\sqrt{\ln (n / N) / N}=O\left(p_{r}\right)$ и $p_{r} e^{-1 / p_{r}}=O\left(1 /\left(N p_{r}\right)\right)$ величины $\rho_{1 r}, \rho_{2 r}$ асимптотически равны при $\lambda=\lambda_{2 r}$, где

$$
\lambda_{2 r}=1+\frac{r}{n}\left(\frac{N c_{2}}{c_{1}}\right)^{2 / 3}(1+o(1)) .
$$


В этом случае при $\lambda<\lambda_{2 r}$ лучшим приближением для распределения $\mu_{r}$ является нормальный закон, при $\lambda>\lambda_{2 r}$ - распределение Пуассона, а если $\lambda=\lambda_{2 r}$, то расстояния между распределением $\mu_{r}$ и приближаючими распределениями асимптотически равны

$$
\rho_{2}=\left(\frac{c_{1} c_{2}^{2}}{N}\right)^{1 / 3}(1+o(1)) .
$$

Ниже приводятся вспомогательные утверждения (леммы 1-3), затем с их помощью будут доказаны теоремы $1-4$ и следствия $1-4$. Введем обозначения:

$$
\begin{array}{cl}
m_{r}=\mathbf{E} \xi_{1}^{(r)}, & \sigma_{r}^{2}=\mathbf{D} \xi_{1}^{(r)}, \quad \alpha_{l}=\mathbf{E} \xi_{1}^{l}, \quad \alpha_{l r}=\mathbf{E}\left(\xi_{1}^{(r)}\right)^{l}, \quad l \geqslant 2, \\
\varphi(t)=\mathbf{E} \exp \left\{i t \xi_{1}\right\}, & \psi(t)=\mathbf{E} \exp \left\{i t \frac{\zeta_{N}-m N}{\sigma \sqrt{N}}\right\}=\exp \left\{-\frac{i t m N}{\sigma \sqrt{N}}\right\} \varphi^{N}\left(\frac{t}{\sigma \sqrt{N}}\right) .
\end{array}
$$

Учитывая (1), (2), нетрудно показать, что

$$
\begin{array}{rlrl}
\alpha_{2} & =\frac{m}{1-\lambda}, & \alpha_{3}=\frac{m(1+\lambda)}{(1-\lambda)^{2}} \\
\alpha_{4} & =\frac{m\left(1+4 \lambda+\lambda^{2}\right)}{(1-\lambda)^{3}}, & \alpha_{5}=\frac{m\left(1+11 \lambda+11 \lambda^{2}+\lambda^{3}\right)}{(1-\lambda)^{4}}, \\
\alpha_{l r} & =\frac{1}{1-p_{r}}\left(\alpha_{l}-r^{l} p_{r}\right), & l=2,3, \ldots, \\
m_{r} & =\frac{1}{1-p_{r}}\left(m-r p_{r}\right), & & \\
\sigma_{r}^{2} & =\frac{1}{1-p_{r}}\left(\frac{m}{1-\lambda}-r^{2} p_{r}\right)-m_{r}^{2}, & & \\
\varphi(t) & =\frac{\ln \left(1-\lambda e^{i t}\right)}{\ln (1-\lambda)} .
\end{array}
$$

Положим

$$
\begin{aligned}
\beta_{1} & =-\frac{\alpha_{3}-3 \alpha_{2} m+2 m^{3}}{6 \sigma^{3} \sqrt{2 N}}, \\
\beta_{2} & =\frac{\alpha_{4}-3 \alpha_{2}^{2}-4 \alpha_{3} m+12 \alpha_{2} m^{2}-6 m^{4}}{8 \sigma^{4} N}, \\
u & =\frac{l-m N}{\sigma \sqrt{N}}, \\
\gamma & = \begin{cases}\frac{1}{n-N}, & \text { если } n / N \rightarrow 1, \\
\frac{\ln (n / N)}{N}, & \text { если } n / N \rightarrow \infty .\end{cases}
\end{aligned}
$$

Лемма 1. Пусть $n, N \rightarrow \infty$ и выполнено одно из следующих условий:

(i) $n / N \rightarrow 1, n-N \rightarrow \infty, r \geqslant 3$;

(ii) $n / N \rightarrow \infty, N / \ln n \rightarrow \infty$.

3 Дискретная математика, т.18 №3 
Тогда прu $e^{u^{2} / 2} \gamma^{2} \rightarrow 0$

$$
\begin{aligned}
\mathbf{P}\left\{\zeta_{N}=l\right\}=\frac{e^{-u^{2} / 2}}{\sigma \sqrt{2 \pi N}}\left(1+\beta_{1}(6 u-\right. & \left.4 u^{3}\right)+\beta_{2}-15 \beta_{1}^{2} \\
& \left.+O\left(e^{u^{2} / 2} \gamma^{2}+\left(u^{2}+u^{6}\right) \gamma+\left(|u|+|u|^{9}\right) \gamma^{3 / 2}\right)\right) .
\end{aligned}
$$

Доказательство. При $n / N \rightarrow 1$ из (7) получаем, что

$$
\lambda=2 \frac{n-N}{N}+o\left(\frac{n-N}{N}\right)
$$

и из (6), (7) следует соотношение

$$
\sigma^{2}=\frac{n-N}{N}(1+o(1))
$$

Если $n / N \rightarrow \infty$, то, как отмечено в [6], уравнение (7) приближенно удовлетворяется при

$$
\lambda=1-\left(\frac{n}{N} \ln \frac{n}{N}\right)^{-1},
$$

и из (6), (7) находим, что

$$
\sigma^{2}=\left(\frac{n}{N}\right)^{2} \ln \frac{n}{N}(1+o(1))
$$

Пусть выполнены условия (i). Учитывая (16), из (13) получаем соотношение

$$
\varphi(t)=e^{i t}\left(1-\frac{1}{\ln (1-\lambda)} \sum_{l=1}^{\infty} \frac{(i t)^{l}}{l !} \sum_{k=1}^{\infty} \frac{(k-1)^{l}}{k} \lambda^{k}\right) .
$$

С помощью равенства (5.2.10) из [7] несложно показать, что при $|t| /(1-\lambda) \leqslant \varepsilon<1$ справедливо неравенство

$$
\sum_{l=6}^{\infty} \frac{(i t)^{l}}{l !} \sum_{k=1}^{\infty} \frac{(k-1)^{l}}{k} \lambda^{k}<\frac{C_{1} \lambda^{2} t^{6}}{(1-\lambda)^{6}}
$$

Отсюда и из (6), (10), (20) получаем равенство

$$
\begin{aligned}
\varphi(t)=e^{i t} & \left(1+i t(m-1)-\frac{t^{2}}{2}\left(\alpha_{2}-2 m+1\right)\right. \\
& -\frac{i t^{3}}{6}\left(\alpha_{3}-3 \alpha_{2}+3 m-1\right)+\frac{t^{4}}{24}\left(\alpha_{4}-4 \alpha_{3}+6 \alpha_{2}-4 m+1\right) \\
& \left.+\frac{i t^{5}}{120}\left(\alpha_{5}-5 \alpha_{4}+10 \alpha_{3}-10 \alpha_{2}+5 m-1\right)+O\left(\frac{m \lambda t^{6}}{(1-\lambda)^{5}}\right)\right) .
\end{aligned}
$$

При $|t| \leqslant(n-N)^{1 / 8}$ из (9) и (21) с помощью формулы Тейлора находим, что

$$
\begin{aligned}
\psi(t)=e^{-t^{2} / 2}\left(1+i \sqrt{2} t^{3} \beta_{1}\right. & +\frac{1}{3} t^{4} \beta_{2}+i t^{5} \beta_{3}-t^{6} \beta_{1}^{2} \\
& \left.+i \frac{\sqrt{2}}{3} t^{7} \beta_{1} \beta_{2}-\frac{\sqrt{2}}{3} t^{9} \beta_{1}^{3}+O\left(\frac{\left(t^{6}+t^{12}\right) \lambda m}{(1-\lambda)^{5} \sigma^{6} N^{2}}\right)\right),
\end{aligned}
$$


где $\beta_{1}, \beta_{2}$ определены в (14),

$$
\beta_{3}=\frac{\alpha_{5}-5 \alpha_{4} m-10 \alpha_{3} \alpha_{2}+20 \alpha_{3} m^{2}+30 \alpha_{2}^{2} m-60 \alpha_{2} m^{3}+24 m^{5}}{5 ! \sigma^{5} N^{3 / 2}} .
$$

Пусть выполнены условия (ii) и $|t| \leqslant C_{2} \sqrt{N / \ln (n / N)}$. Тогда, в силу формулы Тейлора, имеет место равенство

$$
\begin{aligned}
\varphi\left(\frac{t}{\sigma \sqrt{N}}\right)=1+\frac{i m t}{\sigma \sqrt{N}}-\frac{\alpha_{2} t^{2}}{2 \sigma^{2} N} & -\frac{i \alpha_{3} t^{3}}{6 \sigma^{3} N^{3 / 2}} \\
& +\frac{\alpha_{4} t^{4}}{24 \sigma^{4} N^{2}}+\frac{i \alpha_{5} t^{5}}{120 \sigma^{5} N^{5 / 2}}+O\left(\frac{m t^{6}}{(1-\lambda)^{5} \sigma^{6} N^{3}}\right) .
\end{aligned}
$$

Используя (9), (23), как и выше, несложно получить соотношение (22).

По формуле обращения,

$$
\mathbf{P}\left\{\zeta_{N}=l\right\}=\frac{1}{2 \pi \sigma \sqrt{N}} \int_{-\pi \sigma \sqrt{N}}^{\pi \sigma \sqrt{N}} e^{-i u t} \psi(t) d t
$$

и поскольку

$$
\frac{1}{\sqrt{2 \pi}} e^{-u^{2} / 2}=\frac{1}{2 \pi} \int_{-\infty}^{\infty} e^{-i u t} e^{-t^{2} / 2} d t
$$

разность

$$
R_{N}=\sqrt{2 \pi}\left(\sigma \sqrt{2 \pi N} \mathbf{P}\left\{\zeta_{N}=l\right\}-e^{-u^{2} / 2}\right)
$$

можно представить в виде

$$
R_{N}=I_{1}+I_{2}+I_{3}+I_{4}
$$

где

$$
\begin{array}{ll}
I_{1}=\int_{|t| \leqslant A} e^{-i u t}\left(\psi(t)-e^{-t^{2} / 2}\right) d t, & I_{2}=\int_{A<|t| \leqslant \varepsilon \sigma \sqrt{N}} e^{-i u t} \psi(t) d t, \\
I_{3}=\int_{\varepsilon \sigma \sqrt{N}<|t| \leqslant \pi \sigma \sqrt{N}} e^{-i u t} \psi(t) d t, & I_{4}=-\int_{|t|>A} e^{-i u t-t^{2} / 2} d t, \quad A=\gamma^{-1 / 8}
\end{array}
$$

постоянная $\varepsilon>0$ будет выбрана позднее.

Учитывая (6), (7), (10), (14)-(19), несложно показать, что

$$
\beta_{1}=O\left(\gamma^{1 / 2}\right), \quad \beta_{2}=O(\gamma), \quad \beta_{3}=O\left(\gamma^{3 / 2}\right) .
$$

Согласно равенству (2.5.36.5) в [7]

$$
\int_{0}^{\infty} x^{2 k+\delta} e^{-a x^{2}}\left\{\begin{array}{l}
\sin b x \\
\cos b x
\end{array}\right\} d x=\frac{(-1)^{k} \sqrt{\pi}}{(2 \sqrt{a})^{2 k+1+\delta}} e^{-b^{2} / 4 a} H_{2 k+\delta}\left(\frac{b}{2 a}\right),
$$

где

$$
H_{s}(z)=(-1)^{s} e^{z^{2}} \frac{d^{s} e^{-z^{2}}}{d z^{s}}, \quad \delta=\left\{\begin{array}{l}
0 \\
1
\end{array}\right\} .
$$

Легко проверить, что при фиксированных $l$ имеет место соотношение

$$
\int_{A}^{\infty} t^{l} e^{-t^{2} / 2} d t=O\left(A^{-16}\right)
$$


С помощью (22), (24)-(26) находим, что

$$
\begin{aligned}
I_{1}=\sqrt{2 \pi} e^{-u^{2} / 2}\left(\beta_{1}\left(6 u-4 u^{3}\right)+\right. & \beta_{2}-15 \beta_{1}^{2} \\
& \left.+O\left(e^{u^{2} / 2} \gamma^{2}+\left(u^{2}+u^{6}\right) \gamma+\left(|u|+|u|^{9}\right) \gamma^{3 / 2}\right)\right) .
\end{aligned}
$$

Для $I_{4}$ справедлива оценка

$$
I_{4}=o\left(\gamma^{2}\right)
$$

Оценим $I_{2}$. При выполнении условий (i) из (21) получаем, что при $|t| \leqslant \varepsilon$ и достаточно малом $\varepsilon$ выполнено соотношение

$$
|\varphi(t)|^{2} \leqslant 1-t^{2} \sigma^{2} / 2<e^{-t^{2} \sigma^{2} / 2} .
$$

Отсюда и из (9) следует, что

$$
\left|I_{2}\right| \leqslant 2 \int_{A}^{\infty} e^{-t^{2} / 4} d t=o\left(\gamma^{2}\right) .
$$

Пусть выполнены условия (ii). Интеграл $I_{2}$ представим в виде

$$
I_{2}=I_{2}^{(1)}+I_{2}^{(2)}+I_{2}^{(3)},
$$

где области интегрирования слагаемых соответственно равны

$$
\begin{aligned}
& S_{1}=\{A<|t| \leqslant \delta \sqrt{N / \ln (n / N)}\}, \\
& S_{2}=\{\delta \sqrt{N / \ln (n / N)}<|t| \leqslant \min \{N / \ln (n / N), \varepsilon \sigma \sqrt{N}\}\}, \\
& S_{3}=\{N / \ln (n / N)<|t| \leqslant \varepsilon \sigma \sqrt{N}\},
\end{aligned}
$$

причем область $S_{3}$ пуста, если $N / \ln (n / N) \geqslant \varepsilon \sigma \sqrt{N}$, постоянная $\delta>0$ будет выбрана позднее.

При $t \in S_{1}$ из (9), (23) получаем, что

$$
\ln \psi(t)=-\frac{t^{2}}{2}\left(1-i 2 \sqrt{2} \beta_{1} t+O\left(\frac{t^{2} \ln (n / N)}{N}\right)\right),
$$

при этом $\left|t \beta_{1}\right| \leqslant \delta, t^{2} \ln (n / N) / N \leqslant \delta^{2}$. Следовательно, найдется такое $\delta>0$, что $|\psi(t)| \leqslant e^{-t^{2} / 4}$ и

$$
I_{2}^{(1)}=o\left(\frac{\ln ^{2}(n / N)}{N^{2}}\right) .
$$

Пусть $|t| \leqslant \varepsilon$. Учитывая (13), функцию $\varphi(t)$ представим в виде

$$
\varphi(t)=\frac{\ln \left|1-\lambda e^{i t}\right|}{\ln (1-\lambda)}+i \frac{\arg \left(1-\lambda e^{i t}\right)}{\ln (1-\lambda)},
$$

где $\left|\arg \left(1-\lambda e^{i t}\right)\right| \leqslant \pi$. Поскольку

$$
\frac{\ln \left|1-\lambda e^{i t}\right|}{\ln (1-\lambda)}=1+\frac{\ln \left(1+2 \lambda(1-\cos t)(1-\lambda)^{-2}\right)}{2 \ln (1-\lambda)}
$$


и $1-\cos t \geqslant t^{2} / 4$, получаем, что

$$
\frac{\ln \left|1-\lambda e^{i t}\right|}{\ln (1-\lambda)} \leqslant 1-\frac{\ln \left(1+\lambda t^{2} / 2(1-\lambda)^{2}\right)}{-2 \ln (1-\lambda)} .
$$

При $t \in S_{2}$ выполнено соотношение $|t| /(1-\lambda) \sigma \sqrt{N} \geqslant \delta$, и из (33) находим, что

$$
\frac{\ln \left|1-\lambda e^{i t / \sigma \sqrt{N}}\right|}{\ln (1-\lambda)} \leqslant 1-\frac{C_{3}}{-2 \ln (1-\lambda)}
$$

Отсюда и из (9), (18), (32) следует неравенство

$$
|\psi(t)| \leqslant \exp \left\{-\frac{C_{4} N}{\ln (n / N)}\right\}
$$

с помощью которого получаем оценку

$$
I_{2}^{(2)}=o\left(\frac{\ln ^{2}(n / N)}{N^{2}}\right)
$$

Пусть $N / \ln (n / N)<|t| \leqslant \varepsilon \sigma \sqrt{N}$. Учитывая (18), из (33) находим, что

$$
\frac{\ln \left|1-\lambda e^{i t /(\sigma \sqrt{N})}\right|}{\ln (1-\lambda)} \leqslant 1-\frac{\ln (|t| /(1-\lambda) \sigma \sqrt{N})}{-\ln (1-\lambda)}+\frac{C_{5}}{-\ln (1-\lambda)} .
$$

Используя это неравенство и (9), (18), (19), (32), получаем соотношение

$$
|\psi(t)| \leqslant\left(1-\frac{C_{6} \ln (|t| /((1-\lambda) \sigma \sqrt{N}))}{-\ln (1-\lambda)}\right)^{N / 2} \leqslant\left(\frac{|t|}{(1-\lambda) \sigma \sqrt{N}}\right)^{-C_{7} N / \ln (n / N)},
$$

с помощью которого нетрудно показать, что

$$
I_{2}^{(3)}=o\left(\frac{\ln ^{2}(n / N)}{N^{2}}\right)
$$

Отсюда и из (29)-(31), (34) следует оценка

$$
I_{2}=o\left(\gamma^{2}\right)
$$

Пусть $\varepsilon \sigma \sqrt{N}<|t| \leqslant \pi \sigma \sqrt{N}$. При выполнении условий (i), используя (13) и формулу Тейлора, находим, что

$$
\varphi\left(\frac{t}{\sigma \sqrt{N}}\right)=e^{i t}\left(1+\frac{\lambda}{2}\left(e^{i t}-1\right)+o(\lambda)\right)
$$

и с учетом формулы Эйлера получаем соотношение

$$
\left|\varphi\left(\frac{t}{\sigma \sqrt{N}}\right)\right|^{2}=1-\lambda\left(1-\cos \frac{t}{\sigma \sqrt{N}}\right)+o(\lambda) \leqslant 1-C_{8} \lambda
$$


Отсюда и из (9) следует неравенство $|\psi(t)| \leqslant e^{-C_{9} N \lambda}$, и с помощью (15)-(17) находим, что

$$
\left|I_{3}\right| \leqslant C_{10} \sigma \sqrt{N} e^{-C_{9} N \lambda}=o\left(\gamma^{2}\right)
$$

Пусть выполнены условия (ii). Поскольку максимальный шаг распределения $\xi_{1}$ равен 1 , при $\varepsilon<|t| /(\sigma \sqrt{N}) \leqslant \pi$ имеет место неравенство

$$
|\varphi(t / \sigma \sqrt{N})| \leqslant e^{-C_{11}}
$$

Используя это соотношение, (9), (15) и (19), нетрудно показать, что

$$
\left|I_{3}\right| \leqslant C_{12} \sqrt{(\ln (n / N)) / N} e^{-C_{11} N}=o\left(\gamma^{2}\right) .
$$

Отсюда и из (27), (28), (35), (36) получаем утверждение леммы.

Положим

$$
v=\frac{l-m_{r} S}{\sigma_{r} \sqrt{S}}
$$

Лемма 2. Пусть $r \geqslant 3, n, N \rightarrow \infty$ так, что $n / N \rightarrow 1, n-N \rightarrow \infty$, u пусть $S \rightarrow \infty$ так, что $\sigma_{r}^{2} S \rightarrow \infty$. Тогда при $e^{v^{2}} /\left(\sigma_{r}^{2} S\right) \rightarrow 0$

$$
\mathbf{P}\left\{\zeta_{S}^{(r)}=l\right\}=\frac{e^{-v^{2} / 2}}{\sigma_{r} \sqrt{2 \pi S}}\left(1+O\left(\frac{e^{v^{2} / 2}}{\sigma_{r} \sqrt{S}}\right)\right)
$$

Лемма 3. Пусть $n, N \rightarrow \infty$ так, что $n / N, N / \ln n \rightarrow \infty$, и пусть $S \rightarrow \infty$ так, что $S / \ln n \rightarrow \infty$. Тогда при $e^{v^{2}} \ln (n / N) / S \rightarrow 0$

$$
\mathbf{P}\left\{\zeta_{S}^{(r)}=l\right\}=\frac{e^{-v^{2} / 2}}{\sigma_{r} \sqrt{2 \pi S}}\left(1+\beta_{1 r}\left(6 v-4 v^{3}\right)+O\left(\frac{e^{v^{2} / 2} \ln (n / N)}{S}\right)\right)
$$

гde

$$
\beta_{1 r}=-\frac{1}{6 \sigma_{r}^{3} \sqrt{2 S}}\left(\alpha_{3 r}-3 \alpha_{2 r} m_{r}+2 m_{r}^{3}\right)=O\left(\frac{\ln (n / N)}{S}\right) .
$$

Леммы 2, 3 являются уточнением леммы 1 из [3] при выполнении соответствующих условий и доказываются по такой же схеме, по которой доказана лемма 1.

Доказательство теорем 1, 2. Заметим, что из равенств (1), (4),(6), (7), (16), (18) следует, что

$$
p_{r} \rightarrow 0, \quad \beta \rightarrow 0
$$

При выполнении условий теоремы 1 , согласно теореме 2.5.2 из [1], имеет место равенство

$$
\left(\begin{array}{l}
N \\
k
\end{array}\right) p_{r}^{k}\left(1-p_{r}\right)^{N-k}=\Pi\left(k, N p_{r}\right)\left(1+\frac{p_{r}}{2}\left(1-v_{r}^{2}\right)+O\left(p_{r}^{2}\left(1+v_{r}^{4}\right)+\frac{1+\left|v_{r}\right|}{\sqrt{N p_{r}}} p_{r}\right)\right) .
$$


Из леммы 1, учитывая (24) и (15), находим, что

$$
\mathbf{P}\left\{\zeta_{N}=n\right\}=\frac{1}{\sigma \sqrt{2 \pi N}}(1+O(\gamma)) .
$$

С помощью (4), (6), (11), (12) несложно проверить, что

$$
\sigma_{r}^{2}=\frac{\sigma^{2}\left(1-p_{r}-\beta\right)}{1-p_{r}}
$$

и для $k=N p_{r}+v_{r} \sqrt{N p_{r}}$ справедливы равенства

$$
\begin{aligned}
\frac{\left(n-k r-(N-k) m_{r}\right)^{2}}{\sigma_{r}^{2}(N-k)} & =v_{r}^{2} \beta\left(1+O\left(\beta+p_{r}+\frac{\left|v_{r}\right| p_{r}}{\sqrt{N p_{r}}}\right)\right), \\
N-k & =N\left(1-p_{r}+O\left(\frac{\left|v_{r}\right| p_{r}}{\sqrt{N p_{r}}}\right)\right) .
\end{aligned}
$$

Тогда, учитывая (37), (40) и соотношение $\beta v_{r}^{2} \rightarrow 0$, из лемм 2,3 получаем, что

$$
\begin{aligned}
\mathbf{P}\left\{\zeta_{N-k}^{(r)}=n-k r\right\}=\frac{1}{\sigma \sqrt{2 \pi N}}(1 & +\beta\left(1-v_{r}^{2}\right) / 2 \\
& \left.+O\left(\left(p_{r}^{2}+\beta^{2}\right)\left(1+v_{r}^{4}\right)+p_{r} \beta v_{r}^{2}\right)+R_{1}\right),
\end{aligned}
$$

где $R_{1}$ определено в (8). Отсюда и из (3), (38), (39) следует утверждение теоремы 1.

При выполнении условий теоремы 2 из теоремы 2.5.1 в [1] и легко проверяемого для $k=N p_{r}+u_{r} \sigma_{r r} \sqrt{N}$ равенства

$$
\frac{\left(k-N p_{r}\right)^{2}}{N p_{r}\left(1-p_{r}\right)}=\frac{u_{r}^{2}\left(1-p_{r}-\beta\right)}{1-p_{r}}
$$

находим, что

$$
\begin{aligned}
\left(\begin{array}{l}
N \\
k
\end{array}\right) p_{r}^{k}\left(1-p_{r}\right)^{N-k} & =\frac{1}{\sqrt{2 \pi N p_{r}\left(1-p_{r}\right)}} \exp \left\{-\frac{u_{r}^{2}\left(1-p_{r}-\beta\right)}{2\left(1-p_{r}\right)}\right\} \\
& \times\left(1+\frac{3 u_{r}-u_{r}^{3}}{6 \sqrt{N p_{r}}}+O\left(\frac{\beta+p_{r}}{\sqrt{N p_{r}}}\left(\left|u_{r}\right|+\left|u_{r}\right|^{3}\right)+\frac{1+u_{r}^{6}}{N p_{r}}\right)\right) .
\end{aligned}
$$

При $k=N p_{r}+u_{r} \sigma_{r r} \sqrt{N}$ выполнены соотношения

$$
\begin{aligned}
\frac{\left(n-k r-(N-k) m_{r}\right)^{2}}{\sigma_{r}^{2}(N-k)} & =\frac{u_{r}^{2} \beta}{1-p_{r}}+O\left(\frac{\left|u_{r}\right|^{3} \beta p_{r}}{\sqrt{N p_{r}}}\right), \\
N-k & =N\left(1-p_{r}+O\left(\frac{\left|u_{r}\right| p_{r}}{\sqrt{N p_{r}}}\right)\right) .
\end{aligned}
$$

Учитывая эти равенства и условия $\beta u_{r}^{2} \leqslant C<\infty, u_{r}^{6} / N p_{r} \rightarrow 0$, из леммы 2 получаем, что при $n / N \rightarrow 1$

$$
\mathbf{P}\left\{\zeta_{N-k}^{(r)}=n-k r\right\}=\frac{1}{\sigma_{r} \sqrt{2 \pi N\left(1-p_{r}\right)}} \exp \left\{-\frac{u_{r}^{2} \beta}{2\left(1-p_{r}\right)}\right\}\left(1+O\left(\frac{1}{\sqrt{n-N}}\right)\right) .
$$


Аналогично, из леммы 3 находим, что при $n / N \rightarrow \infty$

$$
\begin{aligned}
& \mathbf{P}\left\{\zeta_{N-k}^{(r)}=n-k r\right\}=\frac{1}{\sigma_{r} \sqrt{2 \pi N\left(1-p_{r}\right)}} \exp \left\{-\frac{u_{r}^{2} \beta}{2\left(1-p_{r}\right)}\right\} \\
& \times\left(1+O\left(\frac{\left|u_{r}\right| p_{r}}{\sqrt{N p_{r}}}+\frac{\ln (n / N)}{N}+\frac{\left|u_{r}\right| \sqrt{\beta \ln (n / N)}}{\sqrt{N}}\right)\right) .
\end{aligned}
$$

Из этих соотношений и (3)-(5), (37), (39), (41) получаем утверждение теоремы 2.

Доказательство теоремы 3. Введем обозначение

$$
\bar{v}=\min \left\{\beta^{-1 / 3}, p_{r}^{-1 / 3}\right\}
$$

и представим $\rho_{1 r}$ в виде $\rho_{1 r}=A_{1}+A_{2}$, где

$$
\begin{aligned}
A_{1} & =\sum_{\left|v_{r}\right| \leqslant \bar{v}}\left|\mathbf{P}\left\{\mu_{r}=k\right\}-\Pi\left(k, N p_{r}\right)\right|, \\
A_{2} & =\sum_{\left|v_{r}\right|>\bar{v}}\left|\mathbf{P}\left\{\mu_{r}=k\right\}-\Pi\left(k, N p_{r}\right)\right|, \\
v_{r} & =\frac{k-N p_{r}}{\sqrt{N p_{r}}} .
\end{aligned}
$$

Согласно равенству (2.5.9) в [1], для любого фиксированного $l$

$$
\sum_{k=0}^{\infty}\left|v_{r}\right|^{l} \Pi\left(k, N p_{r}\right)=O(1)
$$

Из теоремы 1 и (42) получаем, что

$$
A_{1}=\frac{\beta+p_{r}}{2} \sum_{\left|v_{r}\right| \leqslant \bar{v}}\left|1-v_{r}^{2}\right| \Pi\left(k, N p_{r}\right)+O\left(\beta^{2}+p_{r}^{2}\right)+R_{1},
$$

где $R_{1}$ определено в (8).

Поскольку $\left(1+v_{r}^{6}\right) /\left(N p_{r}\right) \rightarrow 0$ при $\left|v_{r}\right| \leqslant v^{*}=\min \left\{\bar{v},\left(N p_{r}\right)^{1 / 7}\right\}$, из теоремы 2.5 .3 в [1] находим, что при $\left|v_{r}\right| \leqslant v^{*}$

$$
\Pi\left(k, N p_{r}\right)=N\left(k, N p_{r}, N p_{r}\right)\left(1+\frac{v_{r}^{3}-3 v_{r}}{6 \sqrt{N p_{r}}}+o\left(\frac{1+v_{r}^{6}}{N p_{r}}\right)\right) .
$$

С помощью леммы 2.5 .1 из [1] и соотношений (37) и $N p_{r} \rightarrow \infty$ несложно показать, что

$$
\begin{aligned}
\sum_{\left|v_{r}\right| \leqslant v^{*}}\left|1-v_{r}^{2}\right| N\left(k, N p_{r}, N p_{r}\right) & =\frac{1}{\sqrt{2 \pi}} \int_{-\infty}^{\infty}\left|1-u^{2}\right| e^{-u^{2} / 2} d u+o(1) \\
& =2 c_{1}+o(1) .
\end{aligned}
$$

Если $\left(N p_{r}\right)^{1 / 7}<\bar{v}$, то, учитывая (42) и условие $N p_{r} \rightarrow \infty$, легко увидеть, что

$$
\sum_{v^{*}<\left|v_{r}\right| \leqslant \bar{v}}\left|1-v_{r}^{2}\right| \Pi\left(k, N p_{r}\right)=o(1)
$$


Поскольку

$$
\begin{aligned}
\frac{\left|v_{r}^{3}-3 v_{r}\right|}{\sqrt{N p_{r}}} & <C_{13}\left(N p_{r}\right)^{-1 / 14}, \\
\frac{1+v_{r}^{6}}{N p_{r}} & \leqslant C_{14}\left(N p_{r}\right)^{-1 / 7},
\end{aligned}
$$

используя (45), получаем неравенство

$$
\sum_{\left|v_{r}\right| \leqslant v^{*}}\left|1-v_{r}^{2}\right| N\left(k, N p_{r}, N p_{r}\right)\left(\frac{\left|v_{r}^{3}-3 v_{r}\right|}{6 \sqrt{N p_{r}}}+o\left(\frac{1+v_{r}^{6}}{N p_{r}}\right)\right) \leqslant \frac{C_{15}}{\left(N p_{r}\right)^{1 / 14}} .
$$

Отсюда и из (37), (43)-(46) следует, что

$$
A_{1}=\left(\beta+p_{r}\right) c_{1}+o\left(\beta+p_{r}\right)+R_{1} .
$$

Введем обозначения

$$
P_{l}=\frac{\mathbf{P}\left\{\zeta_{N-l}=n-l r\right\}}{\mathbf{P}\left\{\zeta_{N}=n\right\}}, \quad l=1,2, \ldots
$$

С помощью (3) и равенства

$$
\sum_{k=0}^{N-l}\left(\begin{array}{c}
N-l \\
k
\end{array}\right) p_{r}^{k}\left(1-p_{r}\right)^{N-l-k} \frac{\mathbf{P}\left\{\zeta_{N-l-k}^{(r)}=n-l r-k r\right\}}{\mathbf{P}\left\{\zeta_{N}=n\right\}}=P_{l}
$$

несложно показать, что

$$
\begin{aligned}
\sum_{\left|v_{r}\right|>\bar{v}} \mathbf{P}\left\{\mu_{r}=k\right\} \leqslant \frac{1}{\bar{v}^{4}} & \sum_{k=0}^{N}\left(\frac{k-N p_{r}}{\sqrt{N p_{r}}}\right)^{4} \mathbf{P}\left\{\mu_{r}=k\right\} \\
= & \frac{1}{\bar{v}^{4}}\left(\left(N p_{r}\right)^{2}\left(P_{4}-4 P_{3}+6 P_{2}-4 P_{1}+1\right)+6 N p_{r}\left(P_{3}-2 P_{2}+P_{1}\right)\right. \\
& -6 N p_{r}^{2}\left(P_{4}-2 P_{3}+P_{2}\right)-p_{r}\left(P_{3}(18-12 / N)-12 P_{2}\right) \\
& \left.+p_{r}^{2}\left(P_{4}(11-6 / N)-8 P_{3}\right)-P_{1}\left(4-1 / N p_{r}\right)+7 P_{2}(1+1 / N)\right)
\end{aligned}
$$

Отсюда, используя лемму 1 , находим, что

$$
\sum_{\left|v_{r}\right|>\bar{v}} \mathbf{P}\left\{\mu_{r}=k\right\} \leqslant C_{16} \bar{v}^{-4}=o\left(\beta+p_{r}\right) .
$$

С учетом (42) легко видеть, что

$$
\sum_{\left|v_{r}\right|>\bar{v}} \Pi\left(k, N p_{r}\right) \leqslant C_{17} \bar{v}^{-4}=o\left(\beta+p_{r}\right)
$$

Из этих соотношений получаем оценку

$$
A_{2}=o\left(\beta+p_{r}\right)
$$

Отсюда и из (47) следует утверждение теоремы 3. 
Доказательство теоремы 4. Пусть

$$
\bar{v}=\min \left\{\left(N p_{r}\right)^{1 / 7}, \sqrt{2} \beta^{-1 / 2}, \sqrt{2} p_{r}^{-1 / 2}\right\} .
$$

Представим $\rho_{2 r}$ в виде $\rho_{2 r}=A_{1}+A_{2}$, где

$$
\begin{aligned}
A_{1} & =\sum_{\left|u_{r}\right| \leqslant \bar{v}}\left|\mathbf{P}\left\{\mu_{r}=k\right\}-N\left(k, N p_{r}, N \sigma_{r r}^{2}\right)\right|, \\
A_{2} & =\sum_{\left|u_{r}\right|>\bar{v}}\left|\mathbf{P}\left\{\mu_{r}=k\right\}-N\left(k, N p_{r}, N \sigma_{r r}^{2}\right)\right|, \\
u_{r} & =\frac{k-N p_{r}}{\sigma_{r r} \sqrt{N}} .
\end{aligned}
$$

Используя лемму 2.5 .1 из [1] при замене суммирования интегрированием, равенство

$$
\int_{0}^{\infty} u^{l} e^{-u^{2} / 2} d u=O(1)
$$

справедливое при любом фиксированном $l$, и соотношения (5), (37), $N p_{r} \rightarrow \infty$, несложно показать, что

$$
\begin{gathered}
\sum_{\left|u_{r}\right| \leqslant \bar{v}}\left|3 u_{r}-u_{r}^{3}\right| N\left(k, N p_{r}, N \sigma_{r r}^{2}\right)=\frac{1}{\sqrt{2 \pi}} \int_{-\infty}^{\infty}\left|3 u-u^{3}\right| e^{-u^{2} / 2} d u+o(1)=6 c_{2}+o(1), \\
\sum_{\left|u_{r}\right| \leqslant \bar{v}}\left(1+\left|u_{r}\right|+\left|u_{r}\right|^{3}+u_{r}^{6}\right) N\left(k, N p_{r}, N \sigma_{r r}^{2}\right)=O(1) .
\end{gathered}
$$

Поскольку $\ln (n / N) / N=O\left(1 / N p_{r}\right)$ при $n / N \rightarrow \infty$ и $1 /(n-N)=o\left(1 / N p_{r}\right)$ при $n / N \rightarrow 1$, с помощью теоремы 2 и (48) получаем, что

$$
A_{1}=\frac{c_{2}}{\sqrt{N p_{r}}}(1+o(1)) \text {. }
$$

Аналогично, учитывая равенство

$$
\int_{-\bar{v}}^{\bar{v}}\left(3 u-u^{3}\right) e^{-u^{2} / 2} d u=0
$$

можно показать, что

$$
\sum_{\left|u_{r}\right| \leqslant \bar{v}}\left(\mathbf{P}\left\{\mu_{r}=k\right\}-N\left(k, N p_{r}, N \sigma_{r r}^{2}\right)\right)=o\left(\frac{1}{\sqrt{N p_{r}}}\right) .
$$

Согласно равенству (2.5.4) в [1], для любого фиксированного $l$

$$
\int_{\bar{v}}^{\infty} u^{l} e^{-u^{2} / 2} d u=O\left(\bar{v}^{l-1} e^{-\bar{v}^{2} / 2}\right) .
$$

Используя это соотношение и лемму 2.5.1 из [1], находим, что

$$
\sum_{\left|u_{r}\right|>\bar{v}} N\left(k, N p_{r}, N \sigma_{r r}^{2}\right)=O\left(\frac{e^{-\bar{v}^{2} / 2}}{\bar{v}}+\frac{e^{-\bar{v}^{2} / 2}}{\sqrt{N p_{r}}}\right) .
$$


Из соотношений (50), (51), (5), (37), $N p_{r} \rightarrow \infty$ и леммы 2.5 .3 из [1] получаем, что

$$
\begin{aligned}
\sum_{\left|u_{r}\right|>\bar{v}} \mathbf{P}\left\{\mu_{r}=k\right\}= & \sum_{\left|u_{r}\right|>\bar{v}} N\left(k, N p_{r}, N \sigma_{r r}^{2}\right)+1-\sum_{k=-\infty}^{\infty} N\left(k, N p_{r}, N \sigma_{r r}^{2}\right) \\
& -\sum_{\left|u_{r}\right| \leqslant \bar{v}}\left(\mathbf{P}\left\{\mu_{r}=k\right\}-N\left(k, N p_{r}, N \sigma_{r r}^{2}\right)\right) \\
= & O\left(\frac{e^{-\bar{v}^{2} / 2}}{\bar{v}}\right)+o\left(\frac{1}{\sqrt{N p_{r}}}\right) .
\end{aligned}
$$

Из этого равенства и (51) следует, что

$$
A_{2}=o\left(\sqrt{\beta e^{-1 / \beta}}+\sqrt{p_{r} e^{-1 / p_{r}}}+\frac{1}{\sqrt{N p_{r}}}\right)
$$

поскольку

$$
e^{-\bar{v}^{2} / 2} / \bar{v} \leqslant C_{18}\left(\sqrt{\beta} e^{-1 / \beta}+\sqrt{p_{r}} e^{-1 / p_{r}}+\left(N p_{r}\right)^{-1 / 7} e^{-\left(N p_{r}\right)^{2 / 7} / 2}\right)
$$

Отсюда и из (49) получаем утверждение теоремы 4.

Для доказательства следствий $1-4$ заметим, что при $n / N \rightarrow 1, r \geqslant 3$ из равенств (4), (6), (7), (16), (17) следуют соотношения

$$
p_{r}=o(\beta), \quad \beta \rightarrow 0
$$

и согласно теоремам 3,4 ,

$$
\begin{aligned}
& \rho_{1 r}=c_{1} \beta(1+o(1))+o\left(\frac{1}{\sqrt{n-N}}\right), \\
& \rho_{2 r}=\frac{c_{2}}{\sqrt{N p_{r}}}+o\left(\frac{1}{\sqrt{N p_{r}}}+\sqrt{\beta e^{-1 / \beta}}\right) .
\end{aligned}
$$

При $n / N \rightarrow \infty$ и фиксированных $r$ из (4), (6), (7), (18), (19) получаем, что

$$
\beta=o\left(p_{r}\right)
$$

и из теорем 3,4 следует, что

$$
\begin{aligned}
& \rho_{1 r}=c_{1} p_{r}+o\left(p_{r}+\sqrt{\ln (n / N) / N}\right) \\
& \rho_{2 r}=\frac{c_{2}}{\sqrt{N p_{r}}}+o\left(\frac{1}{\sqrt{N p_{r}}}+\sqrt{p_{r} e^{-1 / p_{r}}}\right) .
\end{aligned}
$$

Из этих соотношений нетрудно получить следствия $1-4$. 


\section{Список литературы}

1. Колчин В. Ф., Севастьянов Б. А., Чистяков В. П., Случайные размещения. Наука, Москва, 1976.

2. Тимашев А. Н., О распределении числа циклов заданной длины в классе подстановок с известным числом циклов. Дискретная математика (2001) 13, №4, 60-72.

3. Черепанова Е. В., Предельные распределения числа циклов заданной длины в случайной подстановке с известным числом циклов. Дискретная математика (2003) 15, №3, 128-144.

4. Колчин В. Ф., Случайные отображсения. Наука, Москва, 1984.

5. Прохоров Ю. В., Асимптотическое поведение биномиального распределения. Успехи матем. наук (1953) 8, №3, 135-142.

6. Колчин В. Ф., Случайные графы. Физматлит, Москва, 2004.

7. Прудников А. П., Брычков Ю. А., Маричев О. И., Интегралы и ряды. Наука, Москва, 1981.

Статья поступила 19.05.2005. 